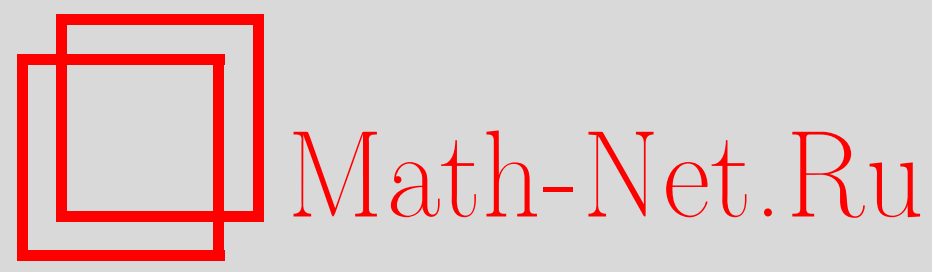

А. А. Грушо, Н. А. Грушо, Е. Е. Тимонина, Теоремы о несуществовании состоятельных последовательностей критериев в некоторых дискретных задачах, Дискрет. матем., 2008, том 20, выпуск 2, 25-31

DOI: https://doi.org/10.4213/dm1001

Использование Общероссийского математического портала Math-Net.Ru подразумевает, что вы прочитали и согласны с пользовательским соглашением http://www . mathnet.ru/rus/agreement

Параметры загрузки:

IP: 54.197 .130 .99

26 апреля 2023 г., 10:37:52 


\title{
Теоремы о несуществовании состоятельных последовательностей критериев в некоторых дискретных задачах
}

\author{
() 2008 г. $\quad$ А. А. Грушо, Н. А. Грушо, Е. Е. Тимонина
}

Несуществование состоятельной последовательности критериев в конечных вероятностных схемах является следствием выполнения некоторых простых достаточных условий, которым должны удовлетворять продолжения вероятностных мер в бесконечных произведениях исходного конечного пространства. Использование этих условий позволяет получить вывод о применимости вероятностных методов для обнаружения отклонения от основной гипотезы.

Работа выполнена при поддержке Российского фонда фундаментальных исследований, проекты 07-01-00484, 07-07-00236.

\section{1. Введение}

Предположим, что в системе, описываемой вероятностными моделями, решение об отклонении системы от нормального поведения может приниматься только на основе статистического решения. Отклонение от нормального поведения характеризуется стохастическим поведением, но уже при других параметрах распределения.

Существует несколько задач, связанных с выявлением изменения параметров, определяющих поведение системы. В некоторых случаях основная задача состоит в том, чтобы как можно быстрее выявить такое изменение параметров для предотвращения сбоя в системе. В других случаях мы можем как угодно долго наблюдать за поведением системы, и выявление изменения параметров распределения наблюдаемых величин может быть основанием для пересмотра архитектуры самой системы. В этом случае возникает задача о возможности разделения нормального и ненормального поведения системы. Однако, для обоснования выводов из результатов такого наблюдения, необходимо знать возможности статистического инструментария по выявлению такого отклонения от нормального поведения. В частности, возникает вопрос, а существует ли статистическая процедура, позволяющая выявить искомое отклонение от нормального поведения. Так как с помощью статистической процедуры редко можно получить ответ “да” или “нет”, а чаще всего ответ звучит “может быть” или “по всей видимости”, в математической статистике стали использовать методы оценки качества статистических процедур. В теории статистических критериев используются понятия наиболее мощный критерий, равномерно наиболее мощный критерий, максиминный критерий, состоятельный критерий.

В данной работе мы рассматриваем такую оценку качества критериев как состоятельность [1]. Невозможность выявления отклонения от нормального поведения характеризуется отсутствием состоятельной последовательности критериев. 
Поскольку данные задачи возникают в задачах, описываемых конечными дискретными моделями, например, при передаче сообщения от одного компьютера к другому, то удается получить простые и понятные достаточные условия несуществования состоятельных последовательностей критериев.

\section{2. Математическая модель}

Пусть $X=\left\{x_{1}, \ldots, x_{m}\right\}$ - конечное множество, которое определяет последовательность конечных множеств $X, X^{2}, \ldots, X^{n}, \ldots$ На каждом из множеств этой последовательности заданы вероятностные меры

$$
\begin{gathered}
\left\{P_{0, n}\left(x_{i_{1}}, \ldots, x_{i_{n}}\right),\left(x_{i_{1}}, \ldots, x_{i_{n}}\right) \in X^{n}\right\}, \\
\left\{P_{1, \theta, n}\left(x_{i_{1}}, \ldots, x_{i_{n}}\right), \theta \in \Theta,\left(x_{i_{1}}, \ldots, x_{i_{n}}\right) \in X^{n}\right\} .
\end{gathered}
$$

Обозначим

$$
X^{\infty}=\left\{\alpha=\left(x_{i_{1}}, x_{i_{2}}, \ldots, x_{i_{n}}, \ldots\right),: x_{i_{n}} \in X,: n=1,2, \ldots\right\}
$$

пространство бесконечных последовательностей. Пусть $\left(x_{i_{1}}, x_{i_{2}}, \ldots, x_{i_{n}}\right) \times X^{\infty}, x_{i_{n}} \in X$, $n=1,2, \ldots,-$ элементарное цилиндрическое множество в $X^{\infty}$, цилиндрическое множество $I_{n}$ есть конечное объединение элементарных цилиндрических множеств, а $\mathscr{A}-$ минимальная $\sigma$-алгебра, порожденная всеми цилиндрическими множествами.

Пусть вероятностные меры, определяемые формулами (1) и (2), являются согласованными семействами конечномерных распределений [2]. Тогда $\left\{P_{0, n}\left(x_{i_{1}}, \ldots, x_{i_{n}}\right)\right\}$ определяет единственную вероятностную меру $P_{0}$ на измеримом пространстве $\left(X^{\infty}, \mathscr{A}\right)$. Для каждого $\theta \in \Theta$ согласованное семейство конечномерных распределений $\left\{P_{1, \theta, n}\left(x_{i_{1}}, \ldots, x_{i_{n}}\right)\right\}$ определяет единственную вероятностную меру $P_{1, \theta}$ на пространстве $\left(X^{\infty}, \mathscr{A}\right)$.

Рассматривается простая гипотеза $H_{0}: P_{0}$ против сложной альтернативы $H_{1}$ : $\left\{P_{1, \theta}, \theta \in \Theta\right\}$. Для задания критерия проверки гипотезы $H_{0}$ против $H_{1}$ на пространстве $\left(X^{\infty}, \mathscr{A}\right)$ при уровне значимости $\alpha$ необходимо определить критическое множество $S \in \mathscr{A}$ такое, что $P_{0}(S)<\alpha$. Мощность данного критерия - это функция $W(\theta)=P_{1, \theta}(S)$ от $\theta \in \Theta$.

Рассмотрим последовательность критериев проверки $H_{0}$ против альтернативы $H_{1}$ с критическими множествами $S_{1}, S_{2}, \ldots$ такую, что

$$
\lim _{k \rightarrow \infty} P_{0}\left(S_{k}\right)=0
$$

Определение 1. Последовательность критериев с критическими множествами $S_{1}, S_{2}, \ldots$ для проверки $H_{0}$ против альтернативы $H_{1}$ называется состоятельной [1], если выполняется условие (3) и для каждого $\theta$

$$
\lim _{k \rightarrow \infty} W_{k}(\theta)=\lim _{k \rightarrow \infty} P_{1, \theta}\left(S_{k}\right)=1,
$$

где $W_{k}(\theta)$ - мощность критерия с критическим множеством $S_{k}$.

Далее в работе исследуется проблема построения достаточных условий, определяющих несуществование состоятельной последовательности критериев. Определяются некоторые минимальные множества альтернатив, в которых задача несуществования состоятельной последовательности критериев имеет важное значение. Для таких минимальных множеств альтернатив строится оценка. Приводятся примеры. 


\section{3. Условия несуществования состоятельной последовательности критериев}

Обозначим через $S_{k}, k=1,2, \ldots$, последовательность критических множеств для критериев, проверяющих гипотезы $H_{0}$ против альтернатив $H_{1}$.

Определение 2. Последовательность критериев для проверки $H_{0}$ против альтернативы $H_{1}$ с критическими множествами $S_{1}, S_{2}, \ldots$ удовлетворяет условию $L$, если существует предел $\lim _{k \rightarrow \infty} S_{k}=S$.

Теорема 1. Пусть для каждого $A \in \mathscr{A}$ такого, что $P_{0}(A)=1$ существует такое $\theta \in \Theta$, что $P_{1, \theta}(A)>0$. Тогда в классе последовательностей тестов, удовлетворяюших условию $L$, не существует состоятельной последовательности критериев.

Доказательство. Предположим противное, и пусть $S_{1}, \ldots, S_{k}, \ldots$ - последовательность критических множеств для состоятельной последовательности критериев в нашей задаче. Согласно условию состоятельности,

$$
\lim _{k \rightarrow \infty} P_{0}\left(S_{k}\right)=0 .
$$

Из условия $L$ следует, что

$$
\lim _{k \rightarrow \infty} P_{0}\left(S_{k}\right)=P_{0}\left(\lim _{k \rightarrow \infty} S_{k}\right)=P_{0}(S) .
$$

Введем обозначение $A=X^{\infty} \backslash S$. Тогда $P_{0}(A)=1-P_{0}(S)$. По определению $A \cap S=\varnothing$.

По условию теоремы существует $\theta \in \Theta$ такое, что

$$
P_{1, \theta}(A)>0 \text {. }
$$

Согласно условию состоятельности,

$$
\lim _{k \rightarrow \infty} P_{1, \theta}\left(S_{k}\right)=1
$$

Из условия $L$ следует, что

$$
\lim _{k \rightarrow \infty} P_{1, \theta}\left(S_{k}\right)=P_{1, \theta}\left(\lim _{k \rightarrow \infty} S_{k}\right)=P_{1, \theta}(S) .
$$

Из определения множества $A$ следует, что $S=X^{\infty} \backslash A$. Поэтому

$$
1=P_{1, \theta}(S)=P_{1, \theta}\left(X^{\infty} \backslash A\right)=1-P_{1, \theta}(A)<1 .
$$

Последнее неравенство противоречит предположению существования состоятельной последовательности критериев, удовлетворяющих условию $L$. Теорема доказана.

Лемма 1. Если существует состоятельная последовательность критериев с критическими множествами $S_{1}, \ldots, S_{k}, \ldots$ для проверки гипотезы $H_{0}$ против альтернативы $H_{1}$, то любая ее подпоследовательность также является состоятельной последовательностью критериев для проверки соответствующей гипотезы против соответствующей альтернативы. 
Доказательство. Поскольку пределы

$$
\lim _{k \rightarrow \infty} P_{0}\left(S_{k}\right)=0, \quad \lim _{k \rightarrow \infty} P_{1, \theta}\left(S_{k}\right)=1,
$$

существуют, они существуют по любой подпоследовательности. Лемма доказана.

Теорема 2. Если существует состоятельная последовательность критериев с критическими множествами $S_{1}, \ldots, S_{k}, \ldots$ для проверки гипотезы $H_{0}$ против альтернативы $H_{1}$, то существует состоятельная последовательность критериев, удовлетворяющая условию $L$.

Доказательство. Пусть $S_{1}, \ldots, S_{k}, \ldots-$ последовательность критических множеств некоторой состоятельной последовательности критериев проверки гипотезы $H_{0}$ против альтернативы $H_{1}$. Пусть $m_{1}, m_{2}, \ldots, m_{k}, \ldots$ - некоторая подпоследовательность последовательности $1,2, \ldots$ Для каждого $r$ рассмотрим события

$$
B_{r}=\bigcup_{k=r}^{\infty} S_{m_{k}} .
$$

При возрастании $r$ последовательность событий $B_{r}$ монотонно не возрастает. Пусть последовательность $m_{k}, k=1,2, \ldots$, такова, что $P_{0}\left(S_{m_{k}}\right) \leqslant(1 / 2)^{k}$. Тогда

$$
P_{0}\left(B_{r}\right)=P_{0}\left(\bigcup_{k=r}^{\infty} S_{m_{k}}\right) \leqslant \sum_{k=r}^{\infty} P_{0}\left(S_{m_{k}}\right) \leqslant(1 / 2)^{r-1} .
$$

Отсюда следует, что

$$
\lim _{r \rightarrow \infty} P_{0}\left(B_{r}\right)=0 .
$$

Так как последовательность $B_{r}$ монотонно не возрастает, существует множество

$$
B=\lim _{r \rightarrow \infty} B_{r}=\bigcap_{r=1}^{\infty} B_{r} .
$$

Из условия состоятельности критериев с критическими множествами $S_{m_{k}}$ получаем оценку $P_{1, \theta}\left(B_{r}\right) \geqslant P_{1, \theta}\left(S_{m_{r}}\right)$, и $\lim _{r \rightarrow \infty} P_{1, \theta}\left(B_{r}\right)=1$ для каждого $\theta$. При этом

$$
\lim _{r \rightarrow \infty} P_{0}\left(B_{r}\right)=P_{0}\left(\lim _{r \rightarrow \infty} B_{r}\right)=P_{0}(B) .
$$

Таким образом, мы построили состоятельную последовательность критериев, удовлетворяющую условию $L$. Теорема доказана.

Теорема 3. Пусть для каждого $A \in A$ mакого, что $P_{0}(A)=1$ существует $\theta \in \Theta$ такое, что $P_{1, \theta}(A)>0$. Тогда не существует состоятельной последовательности критериев для проверки $H_{0}$ против альтернативы $H_{1}$.

Доказательство. Предположим, что существует состоятельная последовательность критериев с критическими множествами $S_{1}, \ldots, S_{k}, \ldots$ для проверки $H_{0}$ против альтернативы $H_{1}$. Тогда по теореме 2 существует состоятельная последовательность критериев, удовлетворяющая условию $L$. Однако по теореме 1 в классе последовательностей критериев, удовлетворяющих условию $L$, не существует состоятельной последовательности критериев. Данное противоречие доказывает теорему. 


\section{4. Условия несуществования состоятельной последовательности критериев в некоторых дискретных задачах}

Пусть задана некоторая подпоследовательность натуральных чисел $n_{1}, n_{2}, \ldots, n_{k}, \ldots$ Для каждого $k, k=1,2, \ldots$, рассматривается задача проверки простой гипотезы $H_{0, n_{k}}: P_{0, n_{k}}$ против сложной альтернативы $H_{1, n_{k}}:\left\{P_{1, \theta, n_{k}}, \theta \in \Theta\right\}$. Последовательность критериев $T_{k}$, $k=1,2, \ldots$, для проверки гипотез $H_{0, n_{k}}$ против $H_{1, n_{k}}$ называется состоятельной, если уровни значимости для этой последовательности критериев стремятся к 0 , а мощности критериев стремятся к 1 при каждом значении параметра $\theta$.

Вероятностные меры (1), (2) можно рассматривать как вероятностные меры на алгебрах цилиндрических подмножеств пространства бесконечных последовательностей, то есть при любом фиксированном $n$

$$
P_{0, n}\left(x_{i_{1}}, \ldots, x_{i_{n}}\right)=P_{0}\left(\left(x_{i_{1}}, x_{i_{2}}, \ldots, x_{i_{n}}\right) \times X^{\infty}\right) .
$$

Аналогичная формула определяет меру на алгебре цилиндрических множеств для $\theta \in \Theta$

$$
P_{1, \theta, n}\left(x_{i_{1}}, \ldots, x_{i_{n}}\right)=P_{1, \theta}\left(\left(x_{i_{1}}, x_{i_{2}}, \ldots, x_{i_{n}}\right) \times X^{\infty}\right) .
$$

Обозначим через $D_{n_{k}}, k=1,2, \ldots$, последовательность критических множеств для критериев, проверяющих гипотезы $H_{0, n_{k}}$ против альтернатив $H_{1, n_{k}}$. Соответствующие уровни значимости обозначим через $\alpha_{n_{k}}, W_{n_{k}}(\theta), k=1,2, \ldots,-$ функции мощности этих критериев.

Теорема 4. Пусть для каждого $A \in A$ такого, что $P_{0}(A)=1$ существует $\theta \in \Theta$ такое, что $P_{1, \theta}(A)>0$. Тогда не существует состоятельной последовательности критериев, проверяющих гипотезы $H_{0, n_{k}}$ против альтернатив $H_{1, n_{k}}$.

Доказательство. Проверка гипотезы $H_{0, n_{k}}$ против альтернативы $H_{1, n_{k}}$ с помощью критерия, основанного на критическом множестве $D_{n_{k}}$ эквивалентна проверке гипотезы $H_{0}$ против альтернативы $H_{1}$ с помощью критерия, основанного на критическом множестве $D_{n_{k}} \times X^{\infty}$. Если выполняются условия теоремы, то последовательность критериев с критическими множествами $D_{n_{k}} \times X^{\infty}, k=1,2, \ldots$, проверки $H_{0}$ против $H_{1}$ не может быть состоятельной последовательностью критериев. Отсюда следует, что исходная последовательность критериев также не является состоятельной. Теорема доказана.

Теорема 5. Пусть

$$
\Theta=X^{\infty}, \quad P_{1, \theta}(\theta)=1 .
$$

Тогда для любой меры $P_{0}$ и множества альтернатив (6) не существует состоятельной последовательности критериев для проверки $H_{0}$ против $H_{1}$.

Доказательство. Пусть $A-$ произвольное измеримое множество такое, что $P_{0}(A)=1$. Тогда $P_{1, \theta}(\theta)=1$ для любого $\theta \in A$. Следовательно, $P_{1, \theta}(A)=1$. Утверждение теоремы следует из теоремы 3.

Пусть теперь $\Theta \subseteq X^{\infty}$ и $|\Theta|<\infty$.

Теорема 6. Если $P_{0}(x)=0$ для любого $x \in X^{\infty}, a P_{1, x}(x)=1$ для любого $x \in \Theta$, $|\Theta|<\infty$, тогда существует состоятельная последовательность критериев для проверки $H_{0}$ против $H_{1}$. 
Доказательство. Объединение одноточечных множеств $\theta$ для всех $\theta \in \Theta$ является замкнутым множеством в Тихоновском произведении [3], так как $|\Theta|<\infty$. Тогда $X^{\infty} \backslash \Theta$ является открытым множеством в Тихоновском произведении. Ясно, что $P_{0}\left(X^{\infty} \backslash \Theta\right)=1$ и $\left(X^{\infty} \backslash \Theta\right) \cap \Theta=\varnothing$. Тогда по теореме 2 из [5] существует состоятельная последовательность критериев для проверки $H_{0, n}$ против $H_{1, n}$. Теорема доказана.

Пример 1. Рассмотрим последовательность сообщений от компьютерной системы $K A$ к компьютерной системе $K B$. Сообщения принадлежат некоторому конечному множеству, а выбор их для передачи подчиняется сложному вероятностному закону, который мы не можем адекватно описать. Пусть промежутки между сообщениями могут принимать значения 0 и 1. Предположим, что длины промежутков между сообщениями распределены независимо друг от друга и принимают значения 0 и 1 с вероятностями, равными 1/2. Мы рассматриваем вероятность последовательности, которая определяется выбором собственно самих сообщений и независимым выбором промежутков между этими сообщениями. Ясно, что вероятность любой такой последовательности сообщений в данной вероятностной схеме равна нулю [4]. Если отклонение описанного нормального поведения состоит в изменении правила формирования длин промежутков между сообщениями, то задача состоит в выявлении такого изменения.

Если промежутки между сообщениями состоят из одних нулей, то по закону больших чисел критерий, основанный на отклонении относительной частоты единиц от $1 / 2$, будет состоятельным при проверке гипотезы $H_{0}^{n}: P_{0, n}$, где $P_{0, n}-$ равновероятное распределение промежутков между осмысленными сообщениями против альтернативы $H_{1}^{n}: P_{1, \overline{0}, n}$, где $\overline{0}$ - нулевая последовательность.

Теоремы 5 и 6 показывают, что несмотря на то, что не существует состоятельной последовательности критериев в широком классе альтернатив, существует подмножество альтернатив, для которых состоятельная последовательность критериев существует. Это объясняет введение следующего определения.

Определение 3. Множество альтернатив $\Theta_{1} \subseteq \Theta$ называется неотличимым для гипотезы $H_{0}$, если выполняются следующие условия:

(1) $\Theta_{1}-$ бесконечное множество;

(2) любое бесконечное подмножество $\Theta_{1}$ образует множество альтернатив, для которого отсутствует состоятельная последовательность критериев проверки $H_{0}$ против $H_{1}$.

Найдем достаточные условия выделения множеств неотличимых альтернатив. Пусть $\widehat{\Theta}$ - бесконечное множество неотличимых для гипотезы $H_{0}$ альтернатив, для которых при любом $\theta \in \widehat{\Theta}$ существует множество $A_{\theta}$ такое, что $P_{0}\left(A_{\theta}\right)=1$ и $P_{1, \theta}\left(A_{\theta}\right)>0$.

Следующая теорема показывает, что $\widehat{\Theta}$ является неотличимым для гипотезы $H_{0}$ множеством альтернатив.

Теорема 7. Любое бесконечное подмножество множества $\widehat{\Theta}$ образует множество альтернатив, для которого отсутствует состоятельная последовательность критериев проверки гипотезы $H_{0}$ против $H_{1}$.

Доказательство. Любое бесконечное подмножество множества $\widehat{\Theta}$ образует множество альтернатив, удовлетворяющих условию теоремы 1. Поэтому из теоремы 1 следует, что для любого такого бесконечного подмножества альтернатив не существует состоятельной последовательности критериев проверки $H_{0}$ против $H_{1}$. Теорема доказана. 


\section{5. Заключение}

Рассматривая задачу о несуществовании состоятельной последовательности критериев в последовательности конечных вероятностных схем, мы вышли в пространство бесконечных последовательностей, на которых определили меры в соответствии с теоремой Колмогорова о продолжении мер. В бесконечномерном пространстве формируются гипотезы, относительно которых исходные гипотезы определяются с помощью проекций вероятностных мер на конечные произведения пространств.

В бесконечномерном пространства сформулированы и доказаны простые достаточные условия отсутствия последовательностей состоятельных критериев для различения введенных гипотез. Эти же достаточные условия справедливы и для последовательностей критериев в конечных вероятностных схемах.

Поскольку не все альтернативы имеют одинаковую значимость в задаче отсутствия состоятельной последовательности критериев, вводится понятие неотличимого множества параметров для основной гипотезы. В рамках бесконечного подмножества альтернатив из этого множества состоятельных последовательностей критериев не существует.

\section{Список литературы}

1. Леман Э., Проверка статистических гипотез. Наука, Москва, 1964.

2. Неве Ж., Математические основы теории вероятностей. Мир, Москва, 1969.

3. Бурбаки Н., Общая топология. Основные структуры. Наука, Москва, 1968.

4. Прохоров Ю. В., Розанов Ю. А., Теория вероятностей. Наука, Москва, 1993.

5. Грушо А. А., Тимонина Е. Е., Некоторые связи между дискретными статистическими задачами и свойствами вероятностных мер на топологических пространствах. Дискретная математика (2006) 18, №4, 128-135.

Статья поступила 6.05.2007. 